\title{
Creating a 'reverse' integrated primary and mental healthcare clinic for those with serious mental illness
}

\author{
Alexandros Maragakis ${ }^{1}$, Ragavan Siddharthan ${ }^{2}$, Jill RachBeisel $^{3}$ and Cassandra Snipes ${ }^{4}$ \\ ${ }^{1}$ Department of Psychology and Counseling, University of Central Arkansas, Conway, AR, USA \\ ${ }^{2}$ Department of Surgery, Oregon Health and Science University, Portland, OR, USA \\ ${ }^{3}$ Department of Psychiatry, University of Maryland School of Medicine, Baltimore, MD, USA \\ ${ }^{4}$ Department of Psychiatry, Henry Ford Health System, Detroit, MI, USA
}

\begin{abstract}
Individuals with serious mental illness (SMI) are more likely to experience preventable medical health issues, such as diabetes, hyperlipidemia, obesity, and cardiovascular disease, than the general population. To further compound this issue, these individuals are less likely to seek preventative medical care. These factors result in higher usage of expensive emergency care, lower quality of care, and lower life expectancy. This manuscript presents literature that examines the health disparities this population experiences, and barriers to accessing primary care. Through the identification of these barriers, we recommend that the field of family medicine work in collaboration with the field of mental health to implement 'reverse' integrated care (RIC) systems, and provide primary care services in the mental health settings. By embedding primary care practitioners in mental health settings, where individuals with SMI are more likely to present for treatment, this population may receive treatment for somatic care by experts. This not only would improve the quality of care received by patients, but would also remove the burden of managing complex somatic care from providers trained in mental health. The rationale for this RIC system, as well as training and policy reforms, are discussed.
\end{abstract}

Key words: healthcare delivery; interdisciplinary teams; primary care; serious mental illness

Received 10 April 2015; revised 24 August 2015; accepted 26 October 2015;

first published online 20 November 2015

\section{Introduction}

A primary goal of healthcare improvement is the provision of enhanced quality of care, while simultaneously lowering overall costs (Rittenhouse and Shortell, 2009). Evidence indicates that many international healthcare systems have focused on reengineering and improving the primary care (PC) setting in order to achieve such goals (Reid et al., 2009). New models of delivery, such as the patient-centered medical home, have

Correspondence to: Alexandros Maragakis, $\mathrm{PhD}$, Department of Psychology and Counseling, University of Central Arkansas, Conway, AR 72035, USA. Email: maragakisa@uca.edu

(C) Cambridge University Press 2015 grown in popularity and are receiving increased attention from researchers and administrators (Nutting et al., 2009; Reid et al., 2009; Crabtree et al., 2010; Jaen et al., 2010). While proposed benefits of current PC reforms for many health issues may impact the vast majority of the global population in a positive way, there are certain populations that may not experience and or benefit from these changes. This lack of benefit may be particularly true for individuals with serious mental illness (SMI).

Epidemiological reports indicate that $4-6.8 \%$ of the global adult population has had an SMI diagnosis, which includes disorders like schizophrenia and bipolar disorder (Kessler et al., 2009). 
Studies consistently find that those with an SMI are more likely to suffer from a disproportionate amount of preventable medical conditions, including cardiovascular disease, diabetes, hypertension, obesity, hyperlipidemia, and a reduction of 25 years in life expectancy (Druss et al., 2002; Kiraly and Gunning, 2008; Viron and Stern, 2010; De Hert et al., 2011).

Studies that have investigated factors that may explain these poor health outcomes have identified many potential causes. First, individuals with SMI are more likely to engage in unhealthy behaviors, such as smoking, lack of exercise, poor diet, than those in the general population (Kiraly and Gunning, 2008). Second, medications that are used to reduce psychiatric symptoms, like atypical antipsychotics, are associated with side effects that cause elevated glucose levels and weight gain, increasing the probability of diabetes and cardiovascular problems (Kiraly and Gunning, 2008). Third, individuals with SMI are less likely to survey and properly identify their somatic health problems and needs, which results in a decreased likelihood of seeking PC services (Oud and Jong, 2009; van Hasselt et al., 2013a). Finally, even when patients with an SMI present to medical providers, they are less likely to receive the proper medications for somatic health issues, and suffer from the phenomenon of 'diagnostic overshadowing' (Graber et al., 2000; Jones et al., 2008).

To compound the significant somatic health issues faced by this population, those with SMI are less likely to engage in PC, and rely on more expensive care (ie, emergency care) (Druss et al., 2002; Kiraly and Gunning, 2008; van Hasselt et al., 2013b). This may be due to the fact that the PC setting, in its current form, is not properly designed to handle the special needs of this population. For example, the high no-show rates of those with SMI increases the likelihood that they would be dismissed from PC clinics. It also hinders the formation of the important interpersonal relationship between patient and provider that is needed to address the array of complex medical issues (Pastore et al., 2013). Primary care providers (PCPs) are often uncomfortable managing complex psychotropic medications (Fraser and Oyama, 2013) and as a result coordination of care can also be problematic for this population. A psychiatrist practicing in a separate location may manage the patient's psychotropic medications and communication between providers regarding treatment plans can be difficult.
Individuals with SMI have reported difficulties handling noisy and crowded waiting areas, and having to purposefully exaggerate symptoms to be taken seriously by PC staff (Lester et al., 2005). They also report that non-attendance is usually due to worsening mental state, or an immediate crisis. However, medical providers usually attribute these behaviors to patients being irrational and erratic (Lester et al., 2005). This view from providers can be particularly problematic, given that many of these individuals do experience crises (eg, benefit hearings, inability to access transportation, cognitive disorganization, or child care) that prevent them from attending appointments.

Furthermore, perception of care from providers in the PC produced mixed results for this population. Some studies indicate that patients, after overcoming the aforementioned barriers, report positive interactions with $\mathrm{PC}$ staff and highly valuing their care (Oud and Jong, 2009). Yet, other studies indicate that patients have concern of discrimination from staff due to their mental illness, and report concern that their needs are not properly addressed within the PC context (Lester et al., 2005; Wheeler et al., 2014).

Due to the various difficulties patients with SMI face within the PC setting, it has been recommended that changes in the organizational structure of how care is delivered for this population is needed (van Hasslet et al., 2013a). We believe that the answers to addressing the PC concerns with this population may lie with a different model of care, 'reverse' integrated care (RIC). As the name may indicate, the RIC model is the reverse of traditional integrated care (IC) settings. In this model, PCPs are co-located in the mental health setting. For this model to effectively work, we believe that there are organizational, training, and policy considerations that would be required. The following sections describe these changes, and how they may impact healthcare for those with SMI. It is important to note that the recommendations in this paper are still in a developmental phase, and methods to evaluate these services are beyond the scope of the current manuscript.

\section{The RIC model}

The field of mental health has come to appreciate that the majority of patients seen in the PC setting 
present with either a primary or comorbid mental health issue (VandenBos and DeLeon, 1988). To capitalize on this opportunity and improve the quality of care patients receive, many have written on the importance of shifting the delivery of mental health services to be provided within the context of the PC setting (O'Donohue et al., 2006; Blount and Miller, 2009; Rozensky, 2012). While this systematic shift, called integrated care, has produced beneficial results in regards to improvements in quality of care and cost savings (Crosson, 2009; Cummings et al., 2009), it may only be appropriate for certain mental health diagnoses (eg, mild to moderate depression, anxiety, insomnia). Others, like SMI diagnoses, still require more in-depth and specialized mental healthcare.

Given that the literature indicates that the current PC context is problematic for this population, and that many with SMI are most regularly in contact with mental health providers (Viron and Stern, 2010), it may be equally as important in regards to improving quality of care and reducing healthcare costs for PCPs to be embedded in mental health clinics. By placing experts in preventative medicine in locations that individuals with SMI are already likely to receive services (ie, the mental health clinic), healthcare systems may be able to reduce the barriers individuals with SMI face, and successfully provide this population the much needed preventative care services it currently lacks.

The RIC model addresses issues, like lack of receiving medications for somatic health concerns, by eliminating fragmented care. This co-locating of both physical and mental health providers would allow for mental health clinics to benefit from promising new practices in the IC setting, like the team huddle and joint appointments.

Another benefit of the RIC model is that it has the potential to alleviate provider burnout and stress. For example, several authors recommend that until better access to PC occurs for those with SMI, the responsibility of the physical care needs are that of the mental health providers (ie, the psychiatrist and psychiatric nurses) (Viron and Stern, 2010; De Hert et al., 2011). However, some mental health providers are reluctant to address somatic concerns, and report that they do not have the time, expertise, or training to properly address those needs (Happell et al., 2012). This burden currently reduces the quality of care patients receive, and leads to providers ignoring issue that may be beyond the scope of their practice. However, studies that investigate IC models indicate that PCPs report higher levels of job satisfaction being a part of a more comprehensive care team (Crosson, 2009). Therefore, an RIC model may also have the same benefits on mental health provider satisfaction and burnout.

Data indicates that settings, particularly the Veteran's Affairs Healthcare System in the United States, that already provide care within an RIC model improve quality of care (Kilbourne et al., 2011) and reduce preventable hospitalizations (Pirraglia et al., 2011) for those with SMI. While these studies do not describe any processes of care or how the multidisciplinary teams operate, they do provide information on whether the RIC model can be successful on multiple levels in improving healthcare for those with SMI.

Although the care pathways described here address challenges faced in outpatient PC with an SMI population, traditional IC remains more appropriate in inpatient hospital settings. For example, a patient with schizophrenia may be admitted to a cardiology telemetry unit due to heart attack. The PC team will be adept at managing the patient's physical presenting problems. However, a behavioral health specialist should be included in such an inpatient setting in order to target behavioral health problems that interfere with the patient's ability to follow through with treatment recommendations (ie, medication non-adherence, substance abuse, tobacco cessation). This example illustrates the need for traditional IC models of care in inpatient settings that treat patients with SMI, but does not eliminate the potential need for the RIC model for general outpatient treatment.

\section{Changes required for success of RIC settings}

For an RIC model to be effective, efficient, and affordable, systematic changes must occur in order to support the new delivery of care. Given that empirical evidence on which specific elements must occur in a healthcare setting for the RIC model to be successful is lacking, many of the recommendations provided are based on elements that contribute to the success of IC models. While new systematic barriers or concerns may arise in 
the creation of RIC models that are not present in traditional IC models, we believe that these recommendations can create the foundation for a functional and useful system of care.

\section{Definition of mental health setting}

RIC may be appropriate in a number of mental health settings, ranging from group practices of either psychologists or psychiatrists, to psychosocial rehabilitation teams, to larger outpatient clinics, to residential settings. Regardless of setting, is it crucial that both therapists (eg, Clinical Psychologists or Licensed Clinical Social Workers) and psychiatrists practice in the same mental health setting, in order for RIC to be successful. Therapists should implement behavioral health interventions, as this is a crucial step in augmenting medication management. For example, modifiable risk factors such as diet, exercise, and tobacco cessation should be addressed when treating SMI (De Hert et al., 2011). In addition, a psychiatrist must be present to consult with PCPs on medication management and drug interactions. Application of RIC is appropriate in a number of mental health settings; however, at a minimum the team should consist of a therapist, a psychiatrist, and a $\mathrm{PCP}$, given there is a large enough census to allow for the PCP to remain consistently productive.

\section{Changes to the mental health setting}

For the RIC model to be successful, PCPs need to have access to similar equipment they would have in the PC setting. This would include devices such as an electrocardiogram machine, glucometers, pulse oximeters, and sphygmomanometers. Access to a phlebotomist and laboratory for blood work analysis would also be beneficial, given that many preventable diseases are assessed through laboratory findings.

With the addition of a PCP, a change in clinic practices and how mental and physical health staff interacts would need to be systematized. For example, in an IC setting, medical providers are trained to use mental health screening questionnaires to detect mental health issues and refer to mental health providers. A similar process would need to occur, where mental health staff is screening for potential medical health concerns that would require the services of a medical provider. Also, additional support staff that are not typical in a mental health clinic (ie, medical assistants) would be required in order to optimize the family medicine provider's efficiency. Without these basic changes in the clinic, an RIC model would not be able to properly integrate physical and mental health services.

A culture of collaboration must also be fostered. There is continued debate in the IC literature about how to best enhance the practice of care teams; however, the mental health setting should be prepared, at the very least, to accommodate changes such as team meetings, on the spot verbal consults, a shared electronic medical record, and shared office space.

\section{Training recommendations}

Mental health providers that provide services in an IC setting require specialized training beyond that of the traditional mental health provider. This usually involves training regarding physical health problems, operating within 15-20 min appointments, and engaging in 'warm hand-offs' (ie, medical providers referring a patient and seeing them the same day). Extra training for medical providers to meet the needs of patients seen in a mental health setting would most likely be required. Given that the clinic practices are different, the function of the PCP would also be different than that of the traditional PC setting.

An important difference in an RIC model is role of the PCP. In the RIC model, the mental health provider (ie, psychiatrist or therapist) would engage in the traditional 'quarterback' role that PCPs typically assume. This shift in roles would occur because the primary concerns of those with SMI are psychiatric in nature. In the RIC model, PCPs would deliver consult services to mental health providers, and collaboratively establish as a team whether more intense somatic care follow-up is required. If more intensive care for somatic needs is required, then the PCP would assume the somatic care of the patient, and continually collaborate with mental health providers to ensure care needs are being met and are being followed through. This evolution of the role of the PCP has been advocated by others in order to meet the care needs of the patient (Crabtree et al., 2010).

Given the high likelihood of individuals with SMI to be placed on some type of psychotropic medication, it is important for PCPs to be familiar with the side effects of these medications, and 
possible interactions with commonly used medications in PC. For example, angiotensinconverting enzyme (ACE) inhibitors such as lisinopril are a mainstay of treatment for hypertension. It is a relatively safe and effective medication but does decrease the elimination of lithium from the body. A population study from 2004 demonstrated higher levels of lithium toxicity in patients who were started on ACE inhibitors (Juurlink et al., 2004). Being cognizant of medication interactions which are not commonly encountered in the PC setting would be essential for the PCP to be effective.

Finally, PCPs must become comfortable operating with a more flexible schedule that can account for no-shows, 'warm hand-offs,' and walk-ins. As indicated earlier, missing appointments due to various reasons is common among clients with SMI. However, unlike the PC setting, mental health clinics are more lenient and less likely to dismiss clients for no-shows. Also, individuals seen at mental health clinics may be more likely to show up when they do not have appointments (eg, they forgot when they had an appointment, an emergency arose). Providers must be able to adapt to having less prep time than traditionally allotted, and function effectively when a patient arrives unannounced or when a 'warm hand-off' is initiated. By preparing PCPs for these changes, transitions into the mental health setting may be easier, and providers will be ready to engage in a variety of problems that they may have not otherwise been trained for.

\section{Policy and research agenda}

For the RIC model to be successful it also requires policy reform and a rigorous research agenda. In regards to policy, nations that do not have single-payer systems or that do not provide reimbursement for IC service may be problematic for clinics to operate effectively and efficiently. For example, in the United States, some states currently prohibit billing physical and mental health services being provided on the same day. This is particularly problematic, because it causes clinics that offer IC services to engage in problematic practices (ie, scheduling clients on separate days for services), in order to receive payment. Other states have completely different billing systems for physical and mental health concerns, making it difficult to navigate and bill through both systems. This places unnecessary burden on clients, and reduces the probability that they will receive the treatments that they require. Therefore, a uniformed payment structure that allows for same day billing, and that promote the use of team approaches is required. Furthermore, time spent collaborating with other members of the care team is currently not reimbursable in many healthcare systems. This collaboration time is crucial to the success of both IC and RIC, and payment systems must be reformed to fiscally support the time professionals dedicate to coordination.

Continued research is also required to assess the RIC model in regards to its effects on patient and provider satisfaction, quality of care, health outcomes, and cost. Some variables, like patient and provider satisfaction, usage of preventative versus emergency care, may be assessed relatively quickly. However, as is being seen with newer models of care (ie, the patient-centered medical home), it may take many years before we can properly answer the questions about how this model influences health outcomes (Jaén et al., 2010). Therefore, it is important to build measurement systems that will allow us to track and evaluate these variables over many years.

Another important aspect of future research must also involve an analysis of processes of care. Research that provides information about which processes of care are crucial for an RIC model to be optimally successful may provide valuable information for systems that wish to set up RIC models of care and maximize cost benefits for individuals and the system. Along with outcomes research, this process research may help systems achieve the goal of providing quality care without taking unnecessary risks.

\section{Conclusion}

The issues faced by individuals with SMI in regards to PC require systematic reform. The current system of PC does not meet the needs of this group, and creates a large gap in quality and access of services. The current healthcare reform has produced an important 'policy window' that allows for some of the major issues hindering the access to PC needs for those with SMI to be addressed (Druss and Bornemann, 2010). By engaging in 
training and system reform, new pathways of providing preventative care in the mental health setting can be created. These new pathways may potentially decrease the barriers faced by individuals with SMI. By engaging in the RIC model, the same cost savings and improvements in quality of care seen in systems that use IC models may be achieved. However, empirical analysis must be conducted to ensure that the RIC model is feasible, affordable, and improves quality of care.

\section{References}

Blount, F.A. and Miller, B.F. 2009: Addressing the workforce crisis in integrated primary care. Journal of Clinical Psychology in Medical Settings 16, 113-19.

Crabtree, B.F., Nutting, P.A., Miller, W.L., Stange, K.C., Stewart, E.E. and Jaén, C.R. 2010: Summary of the National Demonstration Project and recommendations for the patient-centered medical home. Annals of Family Medicine 8 (Suppl 1), S80-90.

Crosson, F.J. 2009: 21st-century health care - the case for integrated delivery systems. The New England Journal of Medicine 361, 1324-25.

Cummings, N.A., Donohue, W.T. and Cummings, J.L. 2009: The financial dimension of integrated behavioral/ primary care. Journal of Clinical Psychology in Medical Settings 16, 31-39.

De Hert, M., Cohen, D., Bobes, J., Cetkovich-Bakmas, M., Leucht, S., Ndetei, D.M. and Correll, C.U. 2011: Physical illness in patients with severe mental disorders. II. Barriers to care, monitoring and treatment guidelines, plus recommendations at the system and individual level. World Psychiatry: Official Journal of the World Psychiatric Association (WPA) 10, 138-51.

Druss, B.G. and Bornemann, T.H. 2010: Improving health and health care for persons with serious mental illness. JAMA 303, 1972-73.

Druss, B., Rosenheck, R., Desai, M.A.M.D. and Perlin, J.B.P. 2002: Quality of preventive medical care for patients with mental disorders. Medical Care 40, 129-36.

Fraser, K. and Oyama, O. 2013: Knowledge of psychotropics and prescribing preferences of family physicians: a preliminary study. Academic Psychiatry 37, 325-28.

Graber, M.A., Bergus, G., Dawson, J.D., Wood, G.B., Levy, B.T. and Levin, I. 2000: Effect of a patient's psychiatric history on physicians' estimation of probability of disease. Journal of General Internal Medicine 15, 204-6.

Happell, B., Scott, D., Platania-Phung, C. and Nankivell, J. 2012: Should we or shouldn't we? Mental health nurses' views on physical health care of mental health consumers. International Journal of Mental Health Nursing 21, 202-10.

Primary Health Care Research \& Development 2016; 17: 421-427
Jaén, C.R., Ferrer, R.L., Miller, W.L., Palmer, R.F., Wood, R., Davila, M. and Stange, K.C. 2010: Patient outcomes at 26 months in the patient-centered medical home National Demonstration Project. Annals of Family Medicine 8 (Suppl 1), S57-67.

Jones, S., Howard, L. and Thornicroft, G. 2008: 'Diagnostic overshadowing': worse physical health care for people with mental illness. Acta Psychiatrica Scandinavica 118, 169-71.

Juurlink, D.N., Mamdani, M.M., Kopp, A., Rochon, P.A., Shulman, K.I. and Redelmeier, D.A. 2004: Drug-induced lithium toxicity in the elderly: a population-based study. Journal of the American Geriatrics Society 52, 794-98.

Kessler, R.C., Aguilar-Gaxiola, S., Alonso, J., Chatterji, S., Lee, S., Ormel, J., Ustun, T.B. and Wang, P.S. 2009: The global burden of mental disorder: an update from the WHO World Mental Health (WMH) surveys. Epidemiologa $e$ Psichiatria Sociale 18, 23-33.

Kilbourne, A.M., Lai, Z., Bowersox, N., Pirraglia, P. and Bauer, M.S. 2011: Does colocated care improve access to cardiometabolic screening for patients with serious mental illness? General Hospital Psychiatry 33, 634-36.

Kilbourne, A.M., Pirraglia, P.A., Lai, Z., Bauer, M.S., Charns, M.P., Greenwald, D., Welsh, D.E., McCarthy, J.F. and Yano, E.M. 2011: Quality of general medical care among patients with serious mental illness: does colocation of services matter? Psychiatric Services 62, 922-28.

Kiraly, B. and Gunning, K. 2008: Primary care issues in patients with mental illness. Am Fam Physician 78, 355-62.

Lester, H., Tritter, J.Q. and Sorohan, H. 2005: Patients' and health professionals' views on primary care for people with serious mental illness: focus group study. BMJ 330, 1122.

Nutting, P.A., Miller, W.L., Crabtree, B.F., Jaen, C.R., Stewart, E.E. and Stange, K.C. 2009: Initial lessons from the first National Demonstration Project on practice transformation to a patient-centered medical home. Annals of Family Medicine 7, 254-60.

O'Donohue, W., Cummings, N., Cucciarre, M., Cummings, J. and Runyan, C. 2006: Integrated behavioral healthcare: a guide for effective action. New York, NY: Prometheus.

Oud, M.J.T. and Jong, B.M. 2009: Somatic diseases in patients with schizophrenia in general practice: their prevalence and health care. BMC Family Practice 10, 32.

Pastore, P., Griswold, K.S., Homish, G.G. and Watkins, R. 2013: Family practice enhancements for patients with severe mental illness. Community Mental Health Journal 49, 172-77.

Pirraglia, P.A., Kilbourne, A.M., Lai, Z., Friedmann, P.D. and O'Toole, T.P. 2011: Colocated general medical care and preventable hospital admissions for veterans with serious mental illness. Psychiatric Services 62, 554-57.

Reid, R.J., Fishman, P.A., Yu, O., Ross, T.R., Tufano, J.T., Soman, M.P. and Larson, E.B. 2009: Patient-centered medical home demonstration: a prospective, quasi-experimental, before and after evaluation. The American Journal of Managed Care 15, e71-87. 
Rittenhouse, D.R. and Shortell, S.M. 2009: The patientcentered medical home: will it stand the test of health reform? JAMA 301, 2038-40.

Rozensky, R.H. 2012: Health care reform: preparing the psychology workforce. Journal of Clinical Psychology in Medical Settings 19, 5-11.

VandenBos, G.R. and DeLeon, P. 1988: The use of psychotherapy to improve physical health. Psychotherapy 125, 335-43.

van Hasselt, F.M., Oud, M.J.T. and Loonen, A.J.M. 2013a: Improvement of care for the physical health of patients with severe mental illness: a qualitative study assessing the view of patients and families. BMC Health Services Research 13, 426. van Hasselt, F.M., Schoor, S.S., Mookhoek, E.J., Brouwers J.R.B.J., Loonen, A.J.M. and Taxis, K. 2013b: Gaps in health care for the somatic health of outpatients with severe mental illness. International Journal of Mental Health Nursing 22, 249-55.

Viron, M.J. and Stern, T.A. 2010: The impact of serious mental illness on health and healthcare. Psychosomatics 51, 458-65.

Wheeler, A., McKenna, B. and Madell, D. 2014: Access to general health care services by a New Zealand population with serious mental illness. Journal of Primary Health Care 6, 7-16. 